\title{
Predictive Value of Combining the Ankle-Brachial Index and SYNTAX Score for the Outcome in STEMI Patients Undergoing Primary Percutaneous Coronary Intervention
}

\author{
KHALED E. HAMADA, M.Sc.; SEHAM F. BADR, M.D.; YASSER H. EL-BARBARY, M.D. and \\ SAMEH S. KHALIL, M.D. \\ The Department of Cardiovascular Medicine, Faculty of Medicine, Tanta University
}

\begin{abstract}
Background: The Synergy between PCI with TAXUS and Cardiac Surgery (SYNTAX) score is effective for prediction of clinical outcome after Percutaneous Coronary Intervention (PCI). However, its predictive ability is low because it reflects only the coronary characterization.

Objective: Evaluation of predictive value of combining the Ankle-Brachial Index (ABI) and SYNTAX score for the outcome in STEMI patients undergoing primary percutaneous coronary intervention.

Methods: A prospective observational cohort study conducted from June 2016 to May 2017 at Cardiovascular Medicine Department, Tanta University Hospitals in Gharbia Governorate, Egypt. The study enrolled 90 consecutive adult patients of both genders who were diagnosed with definite ST-segment elevation myocardial infarction within 12 hours from the time of symptoms onset, and were treated by primary percutaneous coronary intervention. SYNTAX score was calculated based on its results. ABI-SYNTAX score was calculated by first reclassifying the ABI and SYNTAX scores and then adding them. ABI $<0.4$ was classified as $2,0.4$ to 0.9 as $1,>0.9$ to 1.3 as 0 points; and SYNTAX score $<22$ was classified as 0,23 to 32 as 1 , and $>33$ as 2 points. The resulting scores were added. Patients were classified into 3 groups: ABI-SYNTAX score low (0), ABI-SYNTAX score moderate (1 or 2 ), and ABI-SYNTAX score high (3 or 4).
\end{abstract}

Results: Incidence of MACEs was higher among patients with high ABI-SYNTAX score. From the total incidence of MACEs in the study population $(n=17), 12$ were present in group III (70.6\%), 4 in group II $(23.5 \%)$ and 1 in group I (5.9\%). ABI-SYNATX score was more sensitive than SYNTAX score alone for detection of occurrence of MACEs in patients with high score (group III). Incidence of secondary endpoints, defined as stent thrombosis, in stent restenosis, rehospitalization with acute coronary syndrome and target lesion revascularization, was higher in group III.

Correspondence to: Dr. Khaled E. Hamada,

The Department of Cardiovascular Medicine,

Faculty of Medicine, Tanta University
Conclusion: ABI- SYNTAX score is an independent prognostic factor for both in-hospital adverse outcomes, as well as, short-term adverse outcomes among STEMI patients who underwent primary PCI. It improved the ability of the SYNTAX score which assesses the angiographic characteristics and complexity of coronary artery lesions by combining the SYNTAX score with ABI. The predictive value of ABI-Syntax score with respect to MACEs was superior to Syntax score alone.

Key Words: SYNTAX score - Percutaneous coronary intervention-ABI-SYNTAX score-MACEs.

\section{Introduction}

WORLDWIDE, Coronary Artery Disease (CAD), including acute myocardial infarction, is the single most frequent cause of death accounting for $13.2 \%$ of deaths [1]. Several recent studies have demonstrated a fall in acute and long-term mortality following ST-Segment Elevation Myocardial Infarction (STEMI) with greater use of reperfusion therapy, primary Percutaneous Coronary Intervention (primary PCI), modern antithrombotic therapy and secondary prevention treatments [2]

The Synergy between PCI with TAXUS and Cardiac Surgery (SYNTAX) score is a coronary lesion complexity scoring system that is used as a prognostic tool in the short and long-term risk stratification of patients who undergo Percutaneous Coronary Intervention (PCI) [3] . The SYNTAX score reflects only the coronary characterization, so its ability to predict the prognosis of patients who underwent PCI is lower than that of other scoring systems, including the patient's clinical characteristics [4].

The ABI, which is the ratio of systolic pressure at the ankle to that in the arm, is quick and easy to measure and has been used for years in vascular 
practice to diagnose and assess the severity of peripheral artery disease. However, the ABI is also an indicator of generalized atherosclerosis because lower levels have been linked to higher rates of concomitant coronary and cerebrovascular disease, and with the presence of cardiovascular risk factors [я. Also, in population cohort studies in the USA [6] and Europe [7], a low ABI has been related to an increased incidence of mortality (total and cardiovascular), myocardial infarction and stroke. These increased relative risks have been shown to occur independent of baseline cardiovascular disease and risk factors, suggesting that the ABI might have an independent role in predicting cardiovascular events [6,7].

The goal of the present study was to demonstrate the predictive value of combining the ankle-brachial index and SYNTAX score for the prediction of outcome in STEMI patients undergoing primary percutaneous coronary intervention.

\section{Patients and Methods}

The study was conducted as a prospective observational cohort study from June 2016 to May 2017 at Cardiovascular Medicine Department, Tanta University Hospitals in Gharbia Governorate, Egypt. The study included 90 consecutive adult patients of both genders who were diagnosed with definite STEMI within 12 hours from the time of symptoms onset and were treated by primary PCI. An informed consent was taken from all participants.

Patients included in this study fulfilled all of the following criteria of acute myocardial infarction:

- Persistent anginal chest pain lasting for more than 20 minutes.

- ST-segment elevation of $>1 \mathrm{~mm}$ in two or more standard leads or $2 \mathrm{~mm}$ in two or more contiguous precordial leads, or the presence of new left bundle branch block.

- Primary percutaneous intervention with clear angiographic data enough to enable evaluation of the severity of coronary artery disease [8].

\section{Exclusion criteria were:}

- Patients who are unable to remain supine for the duration of the examination are not candidates for an adequate $\mathrm{ABI}$.

- Patients in whom the use of an occlusive sphygmomanometer cuff may worsen the extremity injury.
- Patients with prior history of myocardial infarction.

- Patients with prior history of CABG.

- Patients with prior history of cerebrovascular disease.

All included patients were subjected to detailed history taking and clinical examination including measurement of vital signs and detection of signs of heart failure/hemodynamic instability according to Killip classification [9].

Measurement of Ankle-Brachial Index [10] was done as follows: Brachial systolic pressures of both arms were obtained, and the higher of the 2 values was chosen as the brachial systolic pressure (the difference between them should be less than $10 \mathrm{mmHg}$ ). The brachial pulse was best appreciated on the medial side of the antecubital fossa. The anterior tibial and posterior tibial systolic pressures of the extremity in question were obtained, and the higher of the 2 values was selected as the ankle pressure measurement. The posterior tibial pulse was best appreciated just dorsal and inferior to the medial malleolus. The dorsalis pedis pulse was best appreciated on the dorsum of the foot between the proximal section of the first and second metatarsals, usually above the navicular bone. Dividing the ankle pressure by the brachial artery pressure; the result was the ABI [10].

Surface ECG and venous sampling for laboratory data, including serum cardiac biomarkers (cardiac troponin I and Creatine kinase-MB), complete blood count, lipid profile, random blood sugar, urea and creatinine, were done. Full 2-D and M- mode echocardiographic study in the standard views (parasternal long axis, short axis, apical four and apical two chambers) was done after successful primary PCI using a commercially available machine in our department (Vivid 7, GE Medical System, Horten, Norway) with a 3.5-MHz transducer. Left ventricular ejection fraction was assessed using modified Simpson method according to the guidelines of the American Society of Echocardiography [11].

All included patients were subjected to primary PCI for the Infarct Related Artery (IRA) according to the ESC guidelines. Coronary angiography was performed under local anesthesia from the femoral approach according to the standard technique. Reperfusion success was assessed according to TIMI blood flow grade. The Infarct Related Artery (IRA) was identified according to the culprit lesion 
on the basis of the infarct location on the admission ECG and the angiographic findings (target vessel, lesion characteristics). The choice of stents (baremetal stent or drug-eluting stent) was left to the operator's discretion. The success of primary PCI was defined as achievement of the TIMI flow of the infarct related artery to grade III. The SYNTAX score of patients was calculated by three interventional cardiologists from initial diagnostic angiography of AMI using a computed program available on the SYNTAX score website [12].

ABI-SYNTAX score was calculated by first reclassifying the $\mathrm{ABI}$ and SYNTAX scores and then adding them. ABI $<0.4$ was classified as 2 , 0.4 to 0.9 as $1,>0.9$ to 1.3 as 0 points; and SYNTAX score $<22$ was classified as 0,23 to 32 as 1 , and $>33$ as 2 points. The resulting scores were added. Patients were classified into 3 groups: ABISYNTAX score low (0), ABI-SYNTAX score moderate (1 or 2 ), and ABI-SYNTAX score high (3 or 4).

Follow-up period was 6 months. Follow-up data were obtained through patient interviews (in person or by telephone), their families. The primary end points were Major Adverse Cardiac and Cerebrovascular Events (MACEs), defined as a composite of all-cause death, myocardial infarction, and stroke. The secondary end points were stent thrombosis, in stent restenosis, re-hospitalization with acute coronary syndrome and target lesion revascularization.

Statistical presentation and analysis of the present study was conducted, using the mean, standard deviation and Chi-square test by SPSS V.20. Numerical data was presented as mean and Standard Deviation (SD) and categorical data was presented as number and percentage. Chi-squared test was used for statistical analysis. When the chisquared test was not appropriate, the Monte Carlo Exact test was applied. The level of significance was adopted at $p<0.05$.

Subjects were informed about the purpose and procedure of the study and benefits of sharing in it. Ethical considerations of the study were carried out according to that of Declaration of Helsinki.

\section{Results}

The study sample was divided according to the ABI-SYNTAX score into three groups: ABISYNTAX low $=0(n=32)$, ABI-SYNTAX moderate: 1-2 $(n=31)$, and ABI-SYNTAX score high: 3- $4(n=27)$. Demographic features of the study population are presented in (Table 1).

As regard risk factors, systemic HTN and diabetes were significant risk factors between the study groups. Smoking also showed statistically significant difference between groups. However, dyslipidemia and family history of premature CAD showed no statistically significant difference between our three study groups (Table 2). Regarding left ventricular ejection fraction, it was noticed that it gets poorer across the study groups, with significant $p$-value $<0.001$. Regarding Killip classification, it was found that number of patients with Killip class IV was higher in group III while in group I, number of patients with Killip class I was the highest among groups with significant $p$ value $<0.001$. (Table 3 ).

Biochemical characteristics of the study groups are presented in (Table 4) which showed that regarding pre-procedural serum creatinine level, there was statistically significant difference between the three groups ( $p$-value $<0.001)$ with higher level of serum creatinine was found in group III. However, regarding pre-procedural serum hemoglobin and cardiac troponin I levels, there was no statistically significant difference between the three groups with $p$-values $=0.555$ and 0.515 respectively.

Anterior STEMI was found to be the most common site of infarction in the study sample but it showed no statistical significant difference between groups (Table 5). Most of the patients had Left Anterior Descending (LAD) as their IRA in the three study groups then Right Coronary Artery (RCA) came after as the second most common IRA and it showed no statistically significant difference between the study groups (Table 6).

It was observed that the number of diseased vessels increased among the study groups from group I with low ABI-SYNTAX score to group III with high ABI-SYNTAX score. Group I included 26 patients $(81.25 \%)$ with single vessel disease, 5 patients $(15.63 \%)$ with two-vessel disease and only 1 patient $(3.12 \%)$ with three-vessel disease. Group II included 12 patients $(38.71 \%)$ with single vessel disease, 14 patients $(45.16 \%)$ with two vessel disease and 5 patients $(16.13 \%)$ with three-vessel disease. Group III included 7 patients $(25.92 \%)$ with single vessel disease, 3 patients $(11.11 \%)$ with two-vessel disease and 17 patients (62.96\%) with three-vessel disease. Comparing number of diseased vessels among the studied groups, there was a statistically significant difference between 
the study groups with $p$-value $<0.001$. Regarding procedural steps such as thrombus aspiration, pre dilatation of the lesion and stent utilization, there was no statistical significant difference between the studied groups with $p$-values $0.707,0.106,0.22$ respectively (Table 7).

Regarding stent characters, it was found that only the type of stent was statistically different between the study groups with the observation that Drug Eluting Stents (DESs) were mostly used in group III and Bare Metal Stents (BMSs) were mostly used in group II, $p=0.004$. However, number of stents, stent diameter and stent length didn't show statistically significant difference between the study groups ( $p$-value $0.33,22$ and 11 respectively) (Table 8).

Regarding TIMI flow, it was found that TIMI flow before revascularization was not statistically significant among the studied groups with $p=0.297$. However, it was noticed that TIMI flow after revascularization decreased across studied groups. In group I, 27 patients (84\%) showed TIMI 3 flow, while 5 patients (16\%) showed TIMI flow 2 . In group II, 27 patients (87\%) showed TIMI 3 flow, while 4 patients $(13 \%)$ showed TIMI flow 2 . In group III, 14 patients (52\%) showed TIMI 3 flow, 10 patients (37\%) showed TIMI flow 2, 3 patients (11\%) showed TIMI flow 1. TIMI flow after revascularization showed statistically significant difference with $p<0.001$ (Table 9).

Patients with higher SYNTAX score were present in group III and values progressively decreased in ABI-SYNTAX score low and intermediate groups. Group I ranged from 7-22, group II ranged from 9-35, group III ranged from 23-34 with statistically significant difference between the study groups, measured $p$-value $<0.001$. Also, there was a statistically significant difference between groups $p 1<0.001$ comparing group I and II, $p 2<0.001$ comparing group I and III, and $p 3$ $<0.001$ comparing group II and III. Patients with lower ABI were found in group III and values increased in groups I and II with statistically significant difference between the three study groups. Also, there was a statistically significant difference between groups II \& III ( $p 2<0.001)$, between groups I \& III $(p 3<0.001)$ as shown in (Table 10).

Table (11) shows that there was a significant association between higher ABI-SYNTAX and MACEs during 6 months follow-up. Higher incidence of all-cause mortality, myocardial infarction and stroke was observed in the high ABI-SYNTAX tertile. From the total incidence of MACEs in the study population $(\mathrm{n}=17), 12$ were present in group III $(70.6 \%), 4$ in group II (23.5\%) and 1 in group I (5.9\%). Table (12) shows that that there was a significant association between higher ABISYNTAX and secondary outcome including stent thrombosis, in stent restenosis, re-hospitalization with acute coronary syndrome and target lesion revascularization.

As regard SYNTAX score alone, from the total incidence of MACEs in the study population $(n=17), 7(41.2 \%)$ were present in group of patients high SYNTAX score (>33), $8(47.1 \%)$ were in patients with intermediate SYNTAX score (23-32) and $2(11.8 \%)$ were present in patients with low SYNTAX score $(<22)$ with significant $p=0.002$ (Table 13). ABI-SYNATX score was more sensitive than SYNTAX score alone for detection of occurrence of MACEs in patients with high score (group III). The Receiver Operating Characteristics (ROC) analysis was performed to compare the performance and predictive accuracy of ABI-Syntax, and Syntax score for predicting MACEs during 6 months follow-up. The Areas Under the Curve (AUC) for ABI-Syntax score and Syntax score was 0.807 , and $0.764(p<0.001, p=0.01$, respectively) as illustrated in Fig. (1). The ABI-Syntax score carried the highest sensitivity $84 \%$ in predicting MACEs with cutoff value $>1$, compared with Syntax score that showed sensitivity of $80 \%$ with cutoff value $>20$. So, the predictive value of ABI-SYNTAX score with respect to MACEs was superior to Syntax score alone (Table 14).

The significant relation between ABI-SYNTAX and prediction of outcome (MACEs) continued even after adjustment for other variables affecting MACEs such as: Age, ejection fraction, stent type, stent diameter, and stent length (Table 15).

Table (1): Demographic features of the study population according to Ankle Brachial Index-SYNTAX score tertiles.

\begin{tabular}{|c|c|c|c|c|c|}
\hline \multirow[b]{2}{*}{ Variables } & \multicolumn{3}{|c|}{ ABI-SYNTAX score } & \multirow[b]{2}{*}{$\begin{array}{l}\text { Sig. } \\
\text { test }\end{array}$} & \multirow[b]{2}{*}{$\begin{array}{c}p- \\
\text { value }\end{array}$} \\
\hline & $\begin{array}{c}\text { Group I } \\
\text { Low } \\
(n=32)\end{array}$ & $\begin{array}{c}\text { Group II } \\
\text { Moderate } \\
(\mathrm{n}=31)\end{array}$ & $\begin{array}{c}\text { Group III } \\
\text { High } \\
(n=27)\end{array}$ & & \\
\hline \multicolumn{6}{|l|}{ Age: } \\
\hline Range & $25-77$ & $33-78$ & $30-80$ & $\mathrm{~F}$ & $0.006^{*}$ \\
\hline Mean \pm S.D & $50.9 \pm 10.74$ & $59.2 \pm 10.1$ & $58.3 \pm 11.6$ & 5.52 & \\
\hline \multicolumn{6}{|l|}{ Sex: } \\
\hline Male & $26(36.6 \%)$ & $23(32.4 \%)$ & $22(31.0 \%)$ & $x^{2}$ & 0.731 \\
\hline Female & $6 \quad(31.6 \%)$ & $8 \quad(42.1 \%)$ & $5 \quad(26.3 \%)$ & 0.626 & \\
\hline
\end{tabular}


Table (2): Comparison between the studied groups as regard to risk factors.

\begin{tabular}{|c|c|c|c|c|c|}
\hline \multirow[b]{2}{*}{ Variables } & \multicolumn{3}{|c|}{ ABI-SYNTAX score } & \multirow[b]{2}{*}{$x^{2}$} & \multirow[b]{2}{*}{$\begin{array}{c}p- \\
\text { value }\end{array}$} \\
\hline & $\begin{array}{l}\text { Group I } \\
\text { Low } \\
(\mathrm{n}=32)\end{array}$ & $\begin{array}{l}\text { Group II } \\
\text { Moderate } \\
(\mathrm{n}=31)\end{array}$ & $\begin{array}{c}\text { Group III } \\
\text { High } \\
(n=27)\end{array}$ & & \\
\hline • DM. & $5 \quad(12.2 \%)$ & $14(34.1 \%)$ & $22(53.7 \%)$ & 25.610 & $0.001 *$ \\
\hline - Hypertension & $6 \quad(18.2 \%)$ & $16(48.5 \%)$ & $11(33.3 \%)$ & 7.599 & $0.022 *$ \\
\hline $\begin{array}{l}\text {-Hypercholest } \\
\text { erolemia }\end{array}$ & $19(31.1 \%)$ & $22(36.1 \%)$ & $20(32.8 \%)$ & 1.669 & 0.434 \\
\hline - Smoking & $21(40.4 \%)$ & $16(30.8 \%)$ & $15(28.8 \%)$ & 1.345 & $0.015^{*}$ \\
\hline $\begin{array}{l}\text { - Family } \\
\text { history }\end{array}$ & $8 \quad(25.0 \%)$ & $10(32.3 \%)$ & $8 \quad(29.6 \%)$ & 0.441 & 0.813 \\
\hline
\end{tabular}

*: Statistically significant. $\quad \chi^{2}$ : Chi square test.

Table (3): Comparing the clinical features of the study groups.

\begin{tabular}{|c|c|c|c|c|c|}
\hline \multirow[b]{2}{*}{ Variables } & \multicolumn{3}{|c|}{ ABI-SYNTAX score } & \multirow[b]{2}{*}{$\begin{array}{l}\text { Sig. } \\
\text { test }\end{array}$} & \multirow[b]{2}{*}{$\begin{array}{c}p- \\
\text { value }\end{array}$} \\
\hline & $\begin{array}{c}\text { Group I } \\
\text { Low } \\
(n=32)\end{array}$ & $\begin{array}{c}\text { Group II } \\
\text { Moderate } \\
(\mathrm{n}=31)\end{array}$ & $\begin{array}{c}\text { Group III } \\
\text { High } \\
(\mathrm{n}=27)\end{array}$ & & \\
\hline $\begin{array}{l}-L V E F \%: \\
\text { Mean士S.D. } \\
\text { Range }\end{array}$ & $\begin{array}{l}49.19 \pm 8.33 \\
30-69\end{array}$ & $\begin{array}{l}46.97 \pm 6.993 \\
35-64\end{array}$ & $\begin{array}{l}40.85 \pm 8.59 \\
25-69\end{array}$ & $\begin{array}{l}F \\
8.400\end{array}$ & $0.001 *$ \\
\hline $\begin{array}{l}\text { - Significance } \\
\text { between } \\
\text { groups }\end{array}$ & $\begin{array}{l}p 1 \\
0.273\end{array}$ & $\begin{array}{l}p 2 \\
0.001\end{array}$ & $\begin{array}{l}p^{3} \\
0.005\end{array}$ & & \\
\hline $\begin{array}{l}\text { - Killip class: } \\
\text { I } \\
\text { II } \\
\text { III } \\
\text { IV }\end{array}$ & $\begin{array}{ll}28 & (41.2 \%) \\
4 & (22.2 \%) \\
0 & (0 \%) \\
0 & (0 \%)\end{array}$ & $\begin{array}{ll}28 & (41.2 \%) \\
3 & (16.7 \%) \\
0 & (0 \%) \\
0 & (0 \%)\end{array}$ & $\begin{array}{l}12(17.6 \%) \\
11(61.1 \%) \\
3(100.0 \%) \\
1(100 \%)\end{array}$ & $\begin{array}{l}\chi^{2} \\
22.650\end{array}$ & $\begin{array}{l}\mathrm{MC}_{p} \\
0.001 *\end{array}$ \\
\hline
\end{tabular}

LVEF : Left Ventricular Ejection Fraction.

$* \quad$ : Statistically significant.

х2 : Chi square test. MCP: Monte Carlo test

F : One way Analysis of Variance (ANOVA).

Significance between groups by post Hoc LSD.

$p 1 \quad$ : For comparing between group I and group II.

p2 : For comparing between group II and group III.

p3 : For comparing between group I and group III.

Table (4): Biochemical characteristics of the study groups.

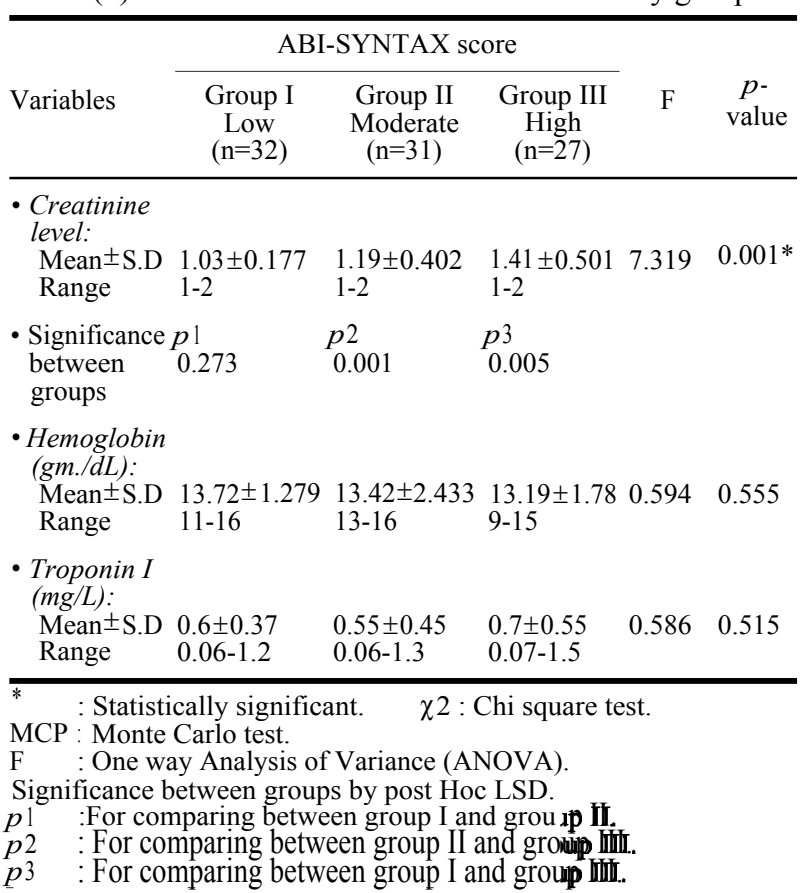

Table (5): Distribution of the studied groups according to the site of infarction.

\begin{tabular}{|c|c|c|c|c|c|}
\hline \multirow[b]{2}{*}{$\begin{array}{l}\text { Site of } \\
\text { infarction }\end{array}$} & \multicolumn{3}{|c|}{ ABI-SYNTAX score } & \multirow[b]{2}{*}{$\chi^{2}$} & \multirow[b]{2}{*}{$\begin{array}{c}p- \\
\text { value }\end{array}$} \\
\hline & $\begin{array}{c}\text { Group I } \\
\text { Low } \\
(n=32)\end{array}$ & $\begin{array}{l}\text { Group II } \\
\text { Moderate } \\
(\mathrm{n}=31)\end{array}$ & $\begin{array}{c}\text { Group III } \\
\text { High } \\
(\mathrm{n}=27)\end{array}$ & & \\
\hline - Anterior & $15(46.87 \%)$ & $19(61.29 .2 \%)$ & $20(74.07 \%)$ & 8.623 & 0.807 \\
\hline • Inferior & $7(21.87 \%)$ & $3(9.68 \%)$ & $2(7.41 \%)$ & & \\
\hline • Lateral & $1(3.12 \%)$ & $2(6.45 \%)$ & $1(3.70 \%)$ & & \\
\hline - Posterior & $1(3.12 \%)$ & $0(0 \%)$ & $0(0 \%)$ & & \\
\hline $\begin{array}{r}\text { - Inferior \& } \\
\text { posterior }\end{array}$ & $2(6.25 \%)$ & $3(9.68 \%)$ & $1(3.70 \%)$ & & \\
\hline $\begin{array}{l}\text { - Inferior \& } \\
\text { right }\end{array}$ & $5(15.62 \%)$ & $3(9.68 \%)$ & $2(7.41 \%)$ & & \\
\hline $\begin{array}{l}\text { - Inferior, } \\
\text { right \& } \\
\text { posterior }\end{array}$ & $1(3.12 \%)$ & $1(3.22 \%)$ & $1(3.70 \%)$ & & \\
\hline
\end{tabular}

*. Statistically significant.

$\chi^{2}$ : Chi square test.

Table (6): Distribution of the studied groups according to the infarct related artery.

\begin{tabular}{|c|c|c|c|c|c|}
\hline \multirow{2}{*}{$\begin{array}{l}\text { Infarct } \\
\text { Related } \\
\text { Artery } \\
\text { (IRA) }\end{array}$} & \multicolumn{3}{|c|}{ ABI-SYNTAX score } & \multirow[b]{2}{*}{$\chi^{2}$} & \multirow[b]{2}{*}{$\begin{array}{c}p- \\
\text { value }\end{array}$} \\
\hline & $\begin{array}{c}\text { Group I } \\
\text { Low } \\
(n=32)\end{array}$ & $\begin{array}{c}\text { Group II } \\
\text { Moderate } \\
(\mathrm{n}=31)\end{array}$ & $\begin{array}{c}\text { Group III } \\
\text { High } \\
(\mathrm{n}=27)\end{array}$ & & \\
\hline LAD & $15(46.9 \%)$ & $19(61.3 \%)$ & $20(74.1 \%)$ & 12.67 & $\mathrm{MC}_{\underline{p}} 0.711$ \\
\hline $\mathrm{RCA}$ & $13(40.6 \%)$ & $6(19.4 \%)$ & $6 \quad(22.2 \%)$ & & \\
\hline LCX & $2(6.2 \%)$ & $6(19.4 \%)$ & $1 \quad(3.7 \%)$ & & \\
\hline OM & $1 \quad(3.1 \%)$ & $0 \quad(0.0 \%)$ & $0 \quad(0.0 \%)$ & & \\
\hline Ramus & $1 \quad(3.1 \%)$ & $\begin{array}{ll}0 & (0.0 \%)\end{array}$ & $\begin{array}{ll}0 & (0.0 \%)\end{array}$ & & \\
\hline
\end{tabular}

LAD : Left Anterior Descending artery.

RCA : Right Coronary Artery

LCX : Left Circumflex Artery.

OM : Obtuse Marginal Artery.

* : Statistically significant.

$\chi^{2} \quad$ : Chi square test.

MCP : Monte Carlo test.

Table (7): Angiographic and procedural characteristics of the study population.

\begin{tabular}{|c|c|c|c|c|c|}
\hline \multirow[b]{2}{*}{ Variables } & \multicolumn{3}{|c|}{ ABI-SYNTAX score } & \multirow[b]{2}{*}{$\chi^{\frac{2}{2}}$} & \\
\hline & $\begin{array}{c}\text { Group I } \\
\text { Low } \\
(n=32)\end{array}$ & $\begin{array}{c}\text { Group II } \\
\text { Moderate } \\
(\mathrm{n}=31)\end{array}$ & $\begin{array}{c}\text { Group III } \\
\text { High } \\
(\mathrm{n}=27)\end{array}$ & & $\begin{array}{c}p \text { - } \\
\text { valuee }\end{array}$ \\
\hline
\end{tabular}

- No. of

affected

vessels:

$126(81.25 \%) \quad 12(38.71 \%) 7 \quad(25.92 \%) 40.757 \quad 0.001^{\text {** }}$

$25 \quad 5 \quad(15.63 \%) \quad 14(45.16 \%) \quad 3 \quad(11.11 \%)$

$3 \quad 1 \quad(3.12 \%) \quad 5 \quad(16.13 \%) \quad 17(62.96 \%)$

- Thrombus $3(9.37 \%) \quad 5 \quad(16.13 \%) 4 \quad(14.81 \%) \quad 0.695 \quad 0.707$ aspiration.

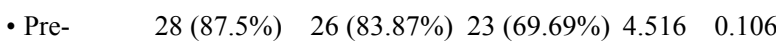
dilatation.

$\begin{array}{llllll}\text { - Stent } & 24(75 \%) & 27(87.09 \%) & 24(88.89 \%) & 12.634 & 0.22\end{array}$

utilization.

No : Number.

* : Statistically significant

$\chi^{2}:$ Chi square test. 
Table (8): Stent characters among study groups.

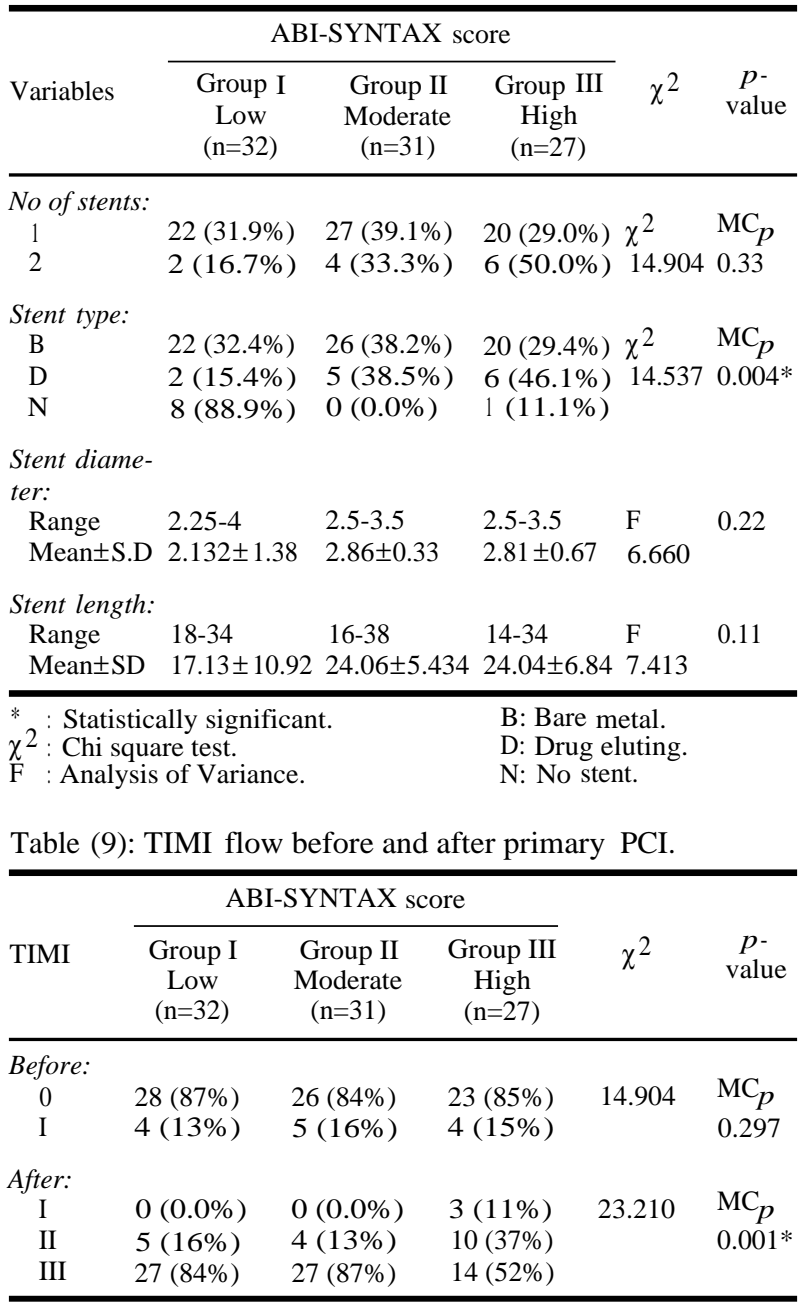

*: Statistically significant.

$\chi 2$ : Chi square test.

Table (10): Comparison between the three studied groups according to Syntax score and ABI.

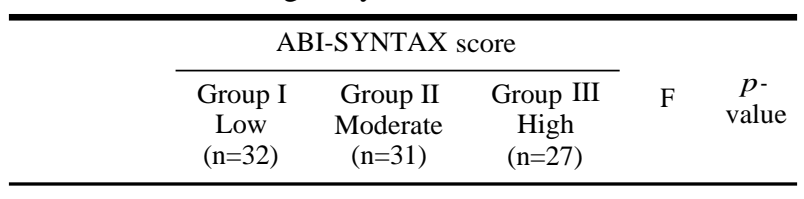

- Syntax

score:

Mean \pm S.D $14.91 \pm 4.1521 .35 \pm 6.053 \quad 30.67 \pm 5.39244 .2910 .001 *$ Range 7-22 9-35 23-34

- Significance $p 1 \quad p 2 \quad p^{3}$ $\begin{array}{llll}\text { between } & 0.001 * & 0.001 * & 0.001 *\end{array}$ groups

$A B I$ :

Mean \pm S.D $1.00 \pm 0.0 \quad 1.00 \pm 0.00 \quad 0.41 \pm 0.50 \quad 66.481 \quad 0.001^{*}$ Range $\quad 1-1 \quad 1-1 \quad 0-1$

- Significance - $\quad p 2 \quad p 3$ between $0.001 * 0.001^{*}$

groups

* : Statistically significant.

F : One way Analysis of Variance (ANOVA).

Significance between groups by post Hoc LSD

$p l \quad$ : For comparing between group I and group II.

$p 2$ : For comparing between group II and group III

$p 3$ : For comparing between group I and group III.

ABI : Ankle-Brachial Index.
Table (11): Primary endpoints and events at 6 months among study groups.

\begin{tabular}{|c|c|c|c|c|c|c|}
\hline \multirow[b]{2}{*}{ Variables } & \multicolumn{4}{|c|}{ ABI-SYNTAX score } & \multirow[b]{2}{*}{$\chi^{2}$} & \multirow[b]{2}{*}{$\begin{array}{c}p- \\
\text { value }\end{array}$} \\
\hline & $\begin{array}{c}\text { Group I } \\
\text { Low } \\
(n=32)\end{array}$ & $\begin{array}{c}\text { Group II } \\
\text { Moderate } \\
(n=31)\end{array}$ & $\begin{array}{c}\text { Group III } \\
\text { High } \\
(n=27)\end{array}$ & $\begin{array}{c}\text { Total } \\
(n=90)\end{array}$ & & \\
\hline $\begin{array}{l}\text { - In hospital } \\
\text { mortality. }\end{array}$ & $\begin{array}{l}1 \\
(3.12 \%)\end{array}$ & $\begin{array}{l}1 \\
(3.22 \%)\end{array}$ & $4(14.8)$ & $6(6.66)$ & 6.204 & $\begin{array}{l}\mathrm{MC}_{p} \\
0.037^{*}\end{array}$ \\
\hline $\begin{array}{l}\text { - Mortality } \\
\text { at } 6 \text { month } \\
\text { follow-up. }\end{array}$ & $0(0 \%)$ & $0(0 \%)$ & $\begin{array}{l}2 \\
(11.1 \%)\end{array}$ & $\begin{array}{l}2 \\
(3.33 \%)\end{array}$ & 17.042 & $\begin{array}{l}\mathrm{MC}_{p} \\
0.001^{*}\end{array}$ \\
\hline $\begin{array}{l}\text { - Re- } \\
\text { infarction. }\end{array}$ & $0(0 \%)$ & $1(3.22 \%)$ & $2(7.4 \%)$ & $\begin{array}{l}3 \\
(3.33 \%)\end{array}$ & 9.484 & $\begin{array}{l}\mathrm{MC}_{p} \\
0.029^{*}\end{array}$ \\
\hline - Stroke. & $0(0 \%)$ & $2(6.44 \%)$ & $4(18.5 \%)$ & $6(6.66)$ & 22.124 & $\mathrm{MC}_{p}$ \\
\hline - MACEs. & $1(5.9)$ & $4(23.5 \%)$ & $12(70.6)$ & $\begin{array}{l}17 \\
(100 \%)\end{array}$ & 17.424 & $\begin{array}{l}0.001 * \\
0.001 *\end{array}$ \\
\hline
\end{tabular}

Table (12): Secondary endpoints and events at 6 months among study groups.

\begin{tabular}{|c|c|c|c|c|c|c|}
\hline \multirow[b]{2}{*}{ Variables } & \multicolumn{4}{|c|}{ ABI-SYNTAX score } & \multirow[b]{2}{*}{$\chi^{2}$} & \multirow[b]{2}{*}{$\begin{array}{c}p- \\
\text { value }\end{array}$} \\
\hline & $\begin{array}{l}\text { Group I } \\
\text { Low } \\
(n=32)\end{array}$ & $\begin{array}{c}\text { Group II } \\
\text { Moderate } \\
(\mathrm{n}=31)\end{array}$ & $\begin{array}{c}\text { Group III } \\
\text { High } \\
(n=27)\end{array}$ & $\begin{array}{c}\text { Total } \\
(\mathrm{n}=90)\end{array}$ & & \\
\hline $\begin{array}{l}\text { - Repeat } \\
\text { unplanned } \\
\text { revascula } \\
\text { rization. }\end{array}$ & $0(0 \%)$ & $\begin{array}{l}4 \\
(12.9 \%)\end{array}$ & $\begin{array}{l}7 \\
(25.9 \%)\end{array}$ & $\begin{array}{l}11 \\
(12.22 \%)\end{array}$ & 20.055 & $\begin{array}{l}\mathrm{MC}_{p} \\
0.001^{*}\end{array}$ \\
\hline $\begin{array}{l}\text { - Re- } \\
\text { hospitaliz } \\
\text { ation. }\end{array}$ & $\begin{array}{l}1 \\
(3.125 \%)\end{array}$ & $\begin{array}{l}8 \\
(25.81 \%)\end{array}$ & $\begin{array}{l}12 \\
(44.44 \%)\end{array}$ & $\begin{array}{l}21 \\
(23.33 \%)\end{array}$ & 26.709 & $\begin{array}{l}\mathrm{MC}_{p} \\
0.001^{*}\end{array}$ \\
\hline $\begin{array}{l}\text { Stent } \\
\text { throm- } \\
\text { bosis. }\end{array}$ & $0(0 \%)$ & $\begin{array}{l}1 \\
(3.22 \%)\end{array}$ & $\begin{array}{l}2 \\
(7.41 \%)\end{array}$ & $\begin{array}{l}3 \\
(3.33 \%)\end{array}$ & 9.484 & $\begin{array}{l}\mathrm{MC}_{p} \\
0.029 *\end{array}$ \\
\hline $\begin{array}{l}\text { In-Stent } \\
\text { Resteno } \\
\text { sis (ISR). }\end{array}$ & $0(0 \%)$ & $\begin{array}{l}3 \\
(9.7 \%)\end{array}$ & $\begin{array}{l}7 \\
(25.9 \%)\end{array}$ & $\begin{array}{l}10 \\
(11.1 \%)\end{array}$ & 25.147 & $\begin{array}{l}\mathrm{MC}_{p} \\
0.001^{*}\end{array}$ \\
\hline
\end{tabular}

* : : Statistically significant.

$\chi^{2}:$ : Chi square test.

$\mathrm{MC}_{p}$ : Mont Carlo for chi square.

Table (13): Distribution of MACEs among according to ABISYNTAX, SYNTAX and ABI scores.

\begin{tabular}{|c|c|c|c|c|c|}
\hline & \multicolumn{3}{|c|}{ ABI-SYNTAX score } & \multirow[b]{2}{*}{$\chi^{2}$} & \multirow[b]{2}{*}{$\begin{array}{c}p- \\
\text { value }\end{array}$} \\
\hline & $\begin{array}{l}\text { Group I } \\
\text { Low } \\
(n=32)\end{array}$ & $\begin{array}{c}\text { Group II } \\
\text { Moderate } \\
(\mathrm{n}=31)\end{array}$ & $\begin{array}{c}\text { Group III } \\
\text { High } \\
(\mathrm{n}=27)\end{array}$ & & \\
\hline \multirow[t]{3}{*}{ MACEs } & $1(5.9 \%)$ & $4(23.5 \%)$ & $12(70.6 \%)$ & 17.424 & $0.001 *$ \\
\hline & \multicolumn{3}{|c|}{ Syntax score } & \multirow{2}{*}{$\chi^{2}$} & \multirow[b]{2}{*}{$p$} \\
\hline & $\begin{array}{c}\text { Low } \\
(n=44)\end{array}$ & $\begin{array}{l}\text { Intermediate } \\
\qquad(\mathrm{n}=29)\end{array}$ & $\begin{array}{l}\text { High } \\
(n=17)\end{array}$ & & \\
\hline MACEs & $2(11.8 \%)$ & $8(47.1 \%)$ & $7(41.2 \%)$ & 12.852 & $0.002 *$ \\
\hline
\end{tabular}


Table (14): Agreement (sensitivity, specificity and accuracy) for ABI-SYNTAX with MACEs.

\begin{tabular}{lccccc}
\hline & Cutoff & AUC & \multicolumn{2}{c}{ Sensitivity } & Specificity \\
\hline ABI-SYNTAX & 1 & 0.807 & $0.001 *$ & $84 \%$ & $52 \%$ \\
Syntax score & 20 & 0.764 & $0.001 *$ & $80 \%$ & $49 \%$ \\
\hline
\end{tabular}

Table (15): Multivariate logistic regression analysis of MACEs.

\begin{tabular}{|c|c|c|c|c|c|c|}
\hline & \multirow{2}{*}{ B } & \multirow{2}{*}{ SE } & \multirow{2}{*}{$p$-value } & \multirow{2}{*}{ OR } & \multicolumn{2}{|c|}{$95 \% \mathrm{CI}$} \\
\hline & & & & & LL & UL \\
\hline ABI-SYNTAX & 0.897 & 0.309 & $0.004 *$ & 2.451 & 1.339 & 4.487 \\
\hline Age & 0.055 & 0.037 & 0.140 & 1.056 & 0.948 & 1.065 \\
\hline $\mathrm{EF}$ & -0.158 & 0.068 & 0.020 & 0.854 & 0.747 & 0.976 \\
\hline DM & -0.545 & 0.839 & 0.516 & 0.580 & 0.112 & 3.005 \\
\hline HTN & 0.723 & 0.740 & 0.328 & 2.062 & 0.484 & 8.788 \\
\hline Stent type & $-9.922-$ & 4.405 & 5.073 & 0.057 & 0.00 & 1.76 \\
\hline Stent diameter & 2.875 & 1.223 & 0.019 & 17.723 & 1.614 & 19.650 \\
\hline Stent length & 0.026 & 0.068 & 0.707 & 1.026 & 0.897 & 1.173 \\
\hline
\end{tabular}

B : Un standardized coefficients.

SE : Standard Error.

OR : Odds Ratio.

CI : Confidence Interval.

LL : Lower Limit.

UL : Upper Limit.

: Statistically significant at $p \leq 0.05$.

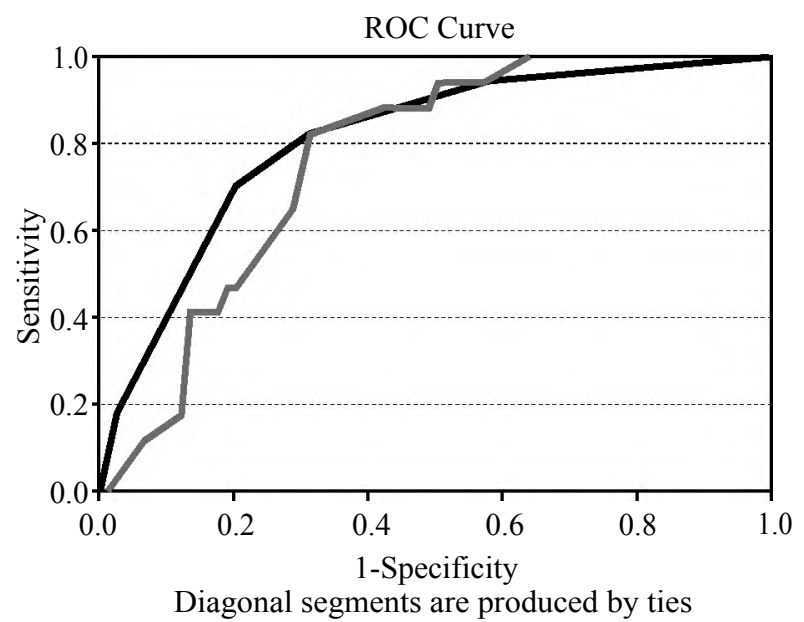

Fig. (1): ROC curve for clinical ABI-SYNTAX score and SYNTAX score.

\section{Discussion}

The first and the only previous study to report that adding the ABI score to the SYNTAX score improved the prediction of MACEs was conducted by Ueki et al., [13] on January 2016 and was done based on the SHINANO registry which was a multicenter, prospective, observational registry designed to compare the differences in baseline characteristics and short-and long-term outcomes after initial PCI between elderly and nonelderly patients. From August 2012 to July 2013, a total of 1,923 consecutive patients with any coronary artery disease (stable angina, ST-segment elevation myocardial infarction, none ST-segment elevation myocardial infarction, and unstable angina) were enrolled from 16 institutions in Nagano prefecture, Japan [13].

In the present study, there was significant correlation between ABI-SYNTAX score and age in studied patients. Patients in group III were older comparing the other two groups. However, there was no significant difference between both genders. As regard risk factors, systemic HTN and diabetes were significant risk factors between the study groups. Smoking also showed statistically significant difference between groups. It was noticed that the number of smokers was lower in the ABISYNTAX high tertile. Smoking was also found to decrease with increasing ABI-SYNTAX score, and it was explained by many different factors. Smoking tendency decreases among the elderly, whereas young patients, despite smoking more than the elderly, have a lower prevalence of comorbidities. Young patients are transferred to the hospital earlier and they do not have diffuse coronary artery disease. Dyslipidemia showed no statistically significant difference between our three study groups. Similarly, Ueki et al., [13] in their study it was found that age was significant in relation to ABISYNTAX groups. Also it was found that dyslipidaemia was not significant in relation to the ABISYNTAX groups. In contrast to the present study, Ueki et al., [13] found that smoking; systemic hypertension and diabetes mellitus have no significant correlation with the ABI-SYNTAX groups. It can be explained by the different sample size in both studies and the prevalence of those risk factors among the study population.

As regard the left ventricular ejection fraction, in the present study, it was found that there is a significant association between higher ABISYNTAX and worsening of left ventricular ejection fraction. It was noticed that it gets poorer across the study groups with mean of $49.19 \pm 8.33 \%, 46.97$ $\pm 6.99 \%$ and $40.85 \pm 8.59 \%$ in the low, intermediate and high groups respectively. Similarly in the study conducted by Ueki et al., [13], lower ejection fractions were recorded in the high ABI-SYNTAX group (with a mean of $57.2 \pm 14.1 \%$ ) as compared to the intermediate and low groups (with a mean of $60.5 \pm 12.5$ and $63.7 \pm 11.6 \%$ respectively). As regard Killip class, the present study demonstrated that higher number of patients in Killip class IV was observed in the high group compared to the intermediate and low groups. 
The present study demonstrated that there was a significant association between higher ABISYNTAX and incidence of MACEs during 6 months follow-up. Higher incidence of all-cause mortality, myocardial infarction and stroke was observed in the high ABI-SYNTAX tertile. From the total incidence of MACEs in the study population $(\mathrm{n}=17), 12$ were present in group III $(70.6 \%)$, 4 in group II $(23.5 \%)$ and 1 in group I $(5.9 \%)$. In the study conducted by Ueki et al., [13], it was found that the incidence of MACE was $6.5 \%$ overall. MACEs, all-cause death, and stroke were significantly higher in the ABI-SYNTAX score high group. However, there were no significant differences in the incidence of cardiac death and myocardial infarction across groups.

As a main result to the present study, it was determined that the ABI- Syntax score carried the highest sensitivity $84 \%$ in predicting MACE with cutoff value $>1$, compared with Syntax score that showed sensitivity of $80 \%$ with cutoff value $>20$. The predictive value of ABI-Syntax score with respect to MACEs was superior to Syntax score alone. The reason behind the improved predictive ability of the ABI-SYNTAX score is that it reflects systemic atherosclerosis better than the SYNTAX score alone.

The present study also demonstrated that there was a significant association between higher ABISYNTAX score and secondary outcome including stent thrombosis, in stent restenosis, rehospitalization with acute coronary syndrome and target lesion revascularization. However, in the study conducted by Ueki et al., [13] there was no significant difference between the study groups as regard target lesion revascularization [13].

In the study conducted by Ueki et al., [13], the ABI-SYNTAX score was an independent predictor of MACEs, whereas the SYNTAX score was not. The Reduction of Atherothrombosis for Continued Health $(\mathrm{REACH})$ registry demonstrated that the 1 -year adverse cardiovascular event rates (cardiovascular death, myocardial infarction, or stroke) increased with the number of symptomatic arterial disease locations, ranging from $2.2 \%$ to $9.2 \%$. Miura et al., [14] also reported that the incidence of MACEs significantly increased with the number of polyvascular diseases, such as peripheral artery disease, carotid artery disease, renal artery disease, and abdominal aorta aneurysm. Although previous studies have found that the SYNTAX score correlated with peripheral artery disease [15] and carotid artery disease [15], the SYNTAX score reflects the complexity of coronary artery disease, not systemic atherosclerosis.
In the present study, the SYNTAX score was an independent short-term predictor of MACEs with $p$-value 0.002 although it demonstrated lower sensitivity compared to the ABI-SYNTAX score. However, in the study conducted by Ueki et al., [13] the SYNTAX score was not a predictor of MACEs [13]. Few studies have reported a correlation between the SYNTAX score and the longterm prognosis of STEMI patients undergoing primary PCI. Akgun T et al., [16] conducted a study of 2993 STEMI patients to investigate the value of the SYNTAX score in predicting prognosis. The investigators reported that a high SYNTAX score is a predictor of all major adverse cardiovascular events in patients with STEMI who have undergone primary PCI. Brown A et al., [17] demonstrated that the SYNTAX score remains an angiographic score and therefore fails to account for clinical parameters that may be important in guiding treatment strategy, but it may has a role in predicting clinical events following primary PCI.

\section{Limitations:}

The study had some limitations. This is a singlecentre experience and represents a limited number of patients. The follow-up period was only 6 months; longer follow-up periods may show different results. Patients weren't administered newgeneration antiplatelet agents such as prasugrel and ticagrelor.

\section{References}

1- WHO| The top 10 causes of death. WHO 2017.http:// www.who. int/mediacentre/factsheets/fs310/en/(accessed 7 Jun2017).

2- MCMANUS D.D., GORE J., YARZEBSKI J., SPENCER F., LESSARD D. and GOLDBERG R.J.: Recent trends in the incidence, treatment, and outcomes of patients with STEMI and NSTEMI. Am. J. Med., 124: 40-7, 2011.

3- CAPODANNO D., CAPRANZANO P., Di SALVO M.E., CAGGEGI A., TOMASELLO D., CINCOTTA G., et al.: Usefulness of SYNTAX Score to Select Patients With Left Main Coronary Artery Disease to Be Treated With Coronary Artery Bypass Graft. J.A.C.C. Cardiovasc. Interv., 2: 731-8, 2009.

4- GARG S., SARNO G., GARCIA-GARCIA H.M., GIRASIS C., WYKRZYKOWSKA J., DAWKINS K.D., et al.: A New Tool for the Risk Stratification of Patients With Complex Coronary Artery Disease: The Clinical SYNTAX Score. Circ. Cardiovasc. Interv., 3: 317-26, 2010.

5- NEWMAN A.B., SHEMANSKI L., MANOLIO T.A., CUSHMAN M., MITTELMARK M., POLAK J.F. et al.: Ankle-arm index as a predictor of cardiovascular disease and mortality in the Cardiovascular Health Study. The Cardiovascular Health Study Group. Arterioscler Thromb. Vasc. Biol., 19: 538-45, 1999. 
6- WEATHERLEY B.D., NELSON J.J., HEISS G., CHAMBLESS L.E., SHARRETT A.R., NIETO F.J., et al.: The association of the ankle-brachial index with incident coronary heart disease: The Atherosclerosis Risk In Communities (ARIC) study, 1987-2001. B.M.C. Cardiovasc. Disord., 7: 3, 2007.

7- HOOI J., KESTER A.D., STOFFERS H.E.J., RINKENS P.E.L., KNOTTNERUS J. and VAN REE J.: Asymptomatic peripheral arterial occlusive disease predicted cardiovascular morbidity and mortality in a 7-year follow-up study. J. Clin. Epidemiol., 57: 294-300, 2004.

8- IBANEZ B., JAMES S., AGEWALL S., ANTUNES M.J., BUCCIARELLI-DUCCI C., BUENO H., et al.: ESC Guidelines for the management of acute myocardial infarction in patients presenting with ST-segment elevation. Eur. Heart J., doi:10.1093/eurheartj/ehx393, 2017.

9- KILLIP T. and KIMBALL J.T.: Treatment of myocardial infarction in a coronary care unit. A two year experience with 250 patients. Am. J. Cardiol., 20: 457-64, 1967.

10- HIRSCH A.T., CRIQUI M.H., TREAT-JACOBSON D., REGENSTEINER J.G., CREAGER M.A., OLIN J.W., et al.: Peripheral arterial disease detection, awareness, and treatment in primary care. J.A.M.A., 286: 1317-24, 2001.

11- LANG R.M., BIERIG M., DEVEREUX R.B., FLACHSKAMPF F.A., FOSTER E., PELLIKKA P.A., et al.: Guidelines Recommendations for chamber quantification. Eur. J. Echocardiogr., 7: 79-108, 2006.

12- SYNTAX Score. http://www.syntaxscore.com/(accessed 25 Oct.2017).
13- UEKI Y., MIURA T., MIYASHITA Y., MOTOKI H., SHIMADA K., KOBAYASHI M., et al.: Predictive Value of Combining the Ankle-Brachial Index and SYNTAX Score for the Prediction of Outcome After Percutaneous Coronary Intervention (from the SHINANO Registry). Am. J. Cardiol., 117: 179-85, 2016.

14- MIURA T., SOGA Y., DOIJIRI T., AIHARA H., YOKOI H., IWABUCHI M., et al.: Prevalence and Clinical Outcome of Polyvascular Atherosclerotic Disease in Patients Undergoing Coronary Intervention. Circ. J., 77: 89-95, 2013.

15- SEBASTIANSKI M., NARASIMHAN S., GRAHAM M.M., TOLEVA O., SHAVADIA J., ABUALNAJA S., et al.: Usefulness of the Ankle-Brachial Index to Predict High Coronary SYNTAX Scores, Myocardium at Risk, and Incomplete Coronary Revascularization. Am. J. Cardiol., 114: 1745-9, 2014

16- AKGUN T., ODUNCU V., BITIGEN A., KARABAY C.Y., ERKOL A., KOCABAY G., et al.: Baseline SYNTAX Score and Long-Term Outcome in Patients With STSegment Elevation Myocardial Infarction Undergoing Primary Percutaneous Coronary Intervention. Clin. Appl. Thromb., 21: 712-9, 2015.

17- BROWN A.J., MCCORMICK L.M., GAJENDRAGADKAR P.R., HOOLE S.P. and WEST N.E.J.: Initial SYNTAX Score Predicts Major Adverse Cardiac Events After Primary Percutaneous Coronary Intervention. Angiology, 65: 408-12, 2014.

\section{الجمع بين مؤشر ضغط دم العضل إلى الكاحل ومؤثر سنتاكس كقيمة تنبؤية المئية

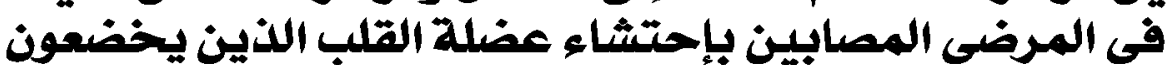 للقسطرة القلبية العلاجية إيجية الآولية}

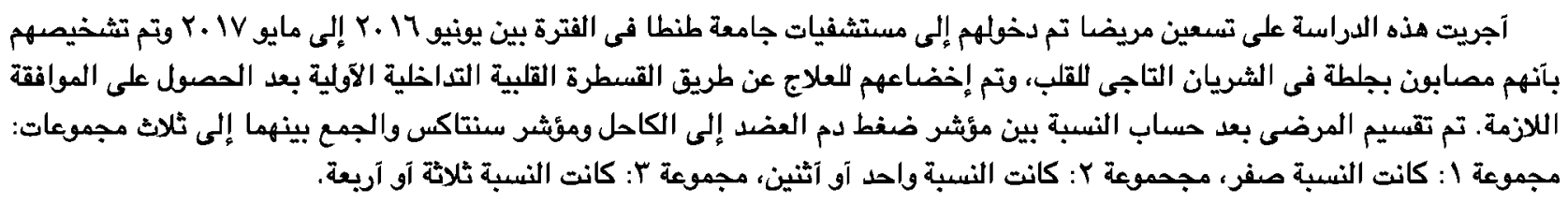

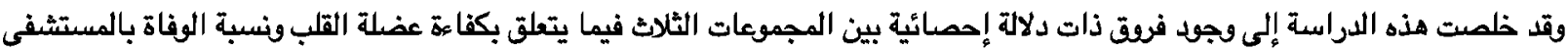

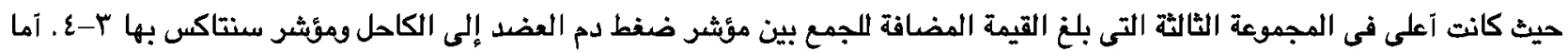
بالنسبة لمضاعفات جلطة الشريان التاجي والتاجي والتى تمت ملاحظتها في المتابعة تريبة المدى في فترة إمتدت ستة آشهر بعد التهد القسطرة

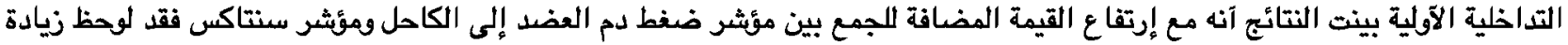

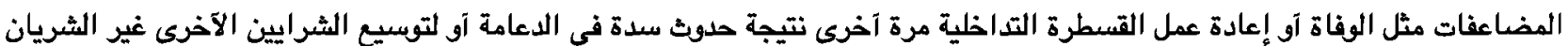

